\title{
A Spontaneous Atypical Femoral Fracture after 2 decades of antiresorptive therapy
}

\author{
Conall Mac Gearailt ${ }^{1}$, Tara McDonnell ${ }^{1}$, Paula O'Shea ${ }^{2}$, and Eamon Mulkerin ${ }^{3}$ \\ ${ }^{1}$ Affiliation not available \\ ${ }^{2}$ University College Hospital \\ ${ }^{3}$ Saolta University Hospital Group
}

June 17, 2020

\begin{abstract}
Prolonged bisphosphonate therapy associated with atypical femoral fracture continues to occur. Protocols to avoid excessive duration of therapy should be utilised routinely. The importance of reviewing risk factors for fractures such as vitamin D status, interacting medications and management of comorbidities such as hemiplegic stroke with spasticity is highlighted.
\end{abstract}

\section{Introduction}

Spontaneous fractures are uncommon and atypical femur fracture (AFF), a spontaneous or low trauma, subtrochanteric or femur shaft stress or insufficiency fracture associated with prolonged antiresorptive therapy, represents the most common manifestation. A 76-year-old woman was admitted from our outpatient clinic with left thigh pain and decreased mobility over 1 month. Whilst transferring from commode to bed on the day after admission, she experienced sudden onset severe left thigh pain and deformity consistent with a spontaneous non-comminuted angulated fracture of left femur. Intramedullary nail fixation of the left femur was performed the following day. She was on Phenobarbitone therapy for 50 years and vitamin D levels were suboptimal while she had previously been receiving Bisphosphonate therapy for 17 years prior to a switch to Denosumab 3 years before presentation. This case clearly demonstrates the spontaneous and atraumatic nature of AFFs following prolonged antiresorptive therapy. Surgical treatment with rehabilitation is required in most cases. A review of therapy with commencement of Teriparatide and enhancement of vitamin D dosage aimed at minimising the risk of further fractures occurred. Reliable mechanisms to avoid protracted antiresorptive therapy beyond recommended guidelines are urgently required.

\section{Case Report}

Spontaneous insufficiency fractures result from physiological stress on weakened bone, with older people particularly susceptible due to osteoporosis (OP), sarcopaenia and neuromotor degeneration [1]. Bisphosphonates are the first-line treatment of established OP, and reduce the likelihood of fractures [2]. However, with prolonged use, AFFs are significant adverse events, and represent the most common manifestation of spontaneous fractures[3]. There is also an association between Denosumab, which replaced Alendronate in this case, and AFFs, albeit less well described [3].

This case highlights the importance of careful monitoring of the duration of antiresorptive therapies and addressing all risk factors to minimise the risk of fractures in at-risk patients.

A 76-year-old lady was admitted from the outpatient clinic(OPD) with a 1-month history of significant left thigh pain and difficulty mobilising. Her past medical history was significant for a stroke with moderate left sided hypertonia, epilepsy, premature menopause, hypothyroidism, OP, hypertension and left sided deep 
vein thrombosis. Her regular medication included levothyroxine, lercanidipine, phenobarbitone, calcium carbonate/cholecalciferol and Denosumab. Previously she was treated with Alendronate for 17 years, switched to Denosumab 3 years ago. She was initially diagnosed with OP on bone densitometry (DEXA scan) in the year 2000 which demonstrated a T score of -2.9 (consistent with severe OP). A fall resulted in a Colles fracture in 2011. Subsequent DEXA scan that year noted worsening OP with a T Score of -3.9 despite therapy. Calcium and vitamin D combination therapy was added with continuation of Alendronate.

On the day after admission, whilst transferring from a commode to her bed, she felt a "snap". Significant left thigh swelling with marked angular deformity was noted on examination. X-Ray imaging revealed an acute angular, transverse, complete fracture of the proximal left femur (Figure 1). Adjusted Calcium was low at $2.13 \mathrm{mmol} / \mathrm{L}(\mathrm{RI}: 2.17-2.51 \mathrm{mmol} / \mathrm{L})$, iPTH was elevated at $97.7 \mathrm{ng} / \mathrm{L}$ (RI:15-65). 25(OH)Vitamin D was suboptimal at $65 \mathrm{nmol} / \mathrm{L}$. Serum Procollagen type I N-terminal propeptide (P1NP): $51 \mu \mathrm{g} / \mathrm{L}$ (RI:16-74);

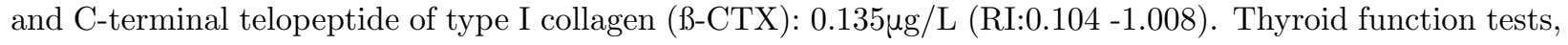
anti-tissue transglutaminase and myeloma screen were normal. X-ray imaging of the right hip revealed no fracture. Left femoral intramedullary nail fixation was successfully performed and she was transferred to the rehabilitation unit where currently she is progressing well. Teriparatide was initiated for bone protection with escalation of Vitamin D dosage. Histological assessment of left femur reamings confirmed the bony tissue to be benign.

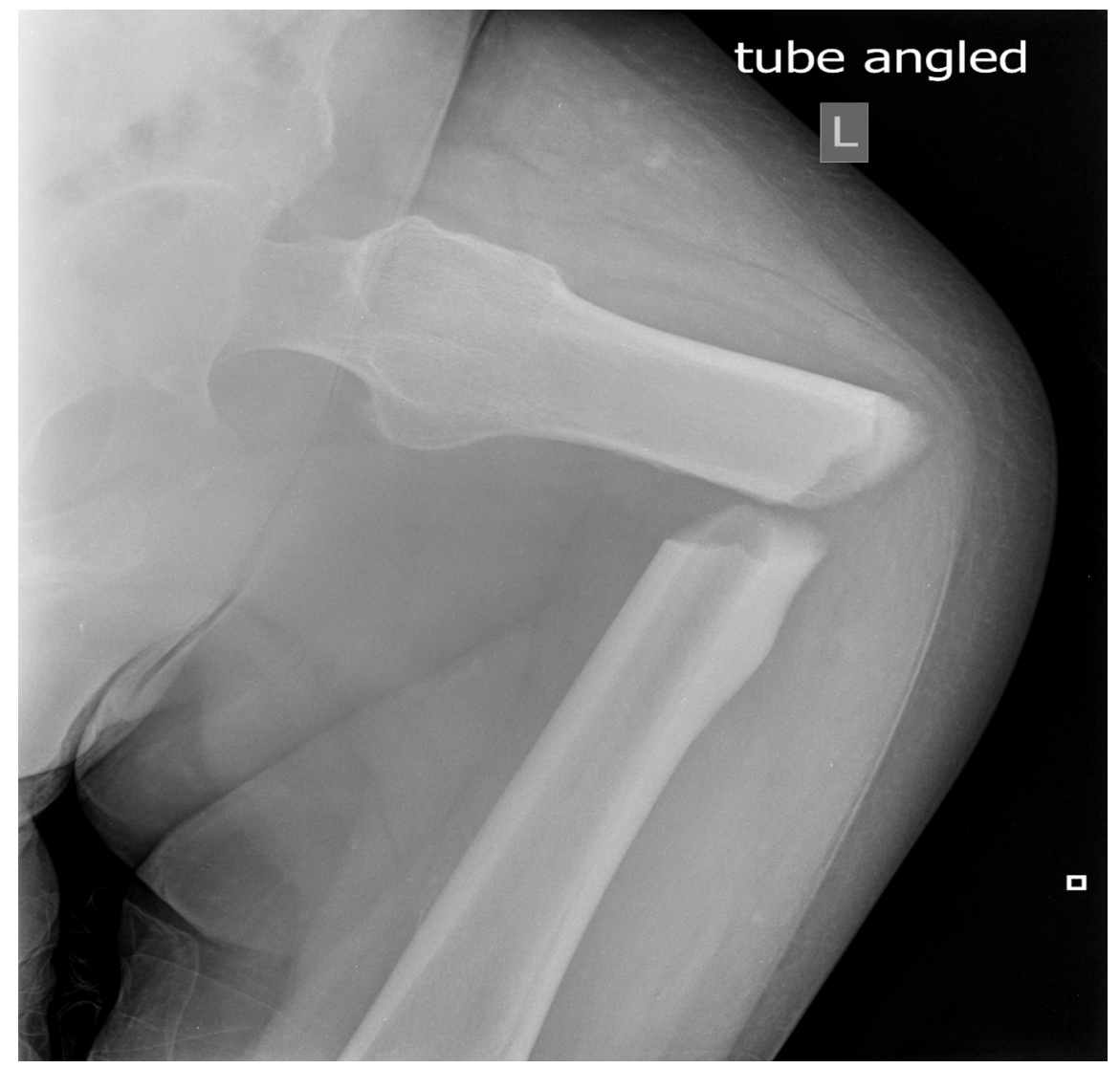

Figure 1. Angulated fracture of upper third of left femur

\section{Discussion}

Osteoporotic fractures cause significant morbidity and mortality as well as healthcare costs. Bisphosphonates, an antiresorptive agent, represent long standing evidence-based therapy for reduction of osteoporotic fractures 
(2). Paradoxically, Bisphosphonates are associated with AFFs (3). As occurred in this case, prodromal thigh or groin pain occurs in the majority of affected patients before a fracture occurs [4]. The fracture site in this lady is classical for an AFF. Upon medication reconciliation, it was apparent that this patient had been treated for OP with Alendronate for 17 consecutive years without any drug pause. Despite having stopped bisphosphonates 3 years prior to admission, this prolonged course likely contributed to her fracture risk. Replacement with Denosumab, which has also been linked with AFFs, was also a contributory factor [5], although the link between AFFs and Bisphosphonates is better established [3].

The mechanism of the paradoxical development of AFFs following prolonged therapy with antiresorptive agents is ill understood. These fractures are unusual in their transverse nature, lack of comminution and spontaneous nature which may suggest a brittle tensile failure mechanism. Multiple material factors and geometric factors may contribute to the development of this fracture subtype [6] including the potential for the geometry of the upper femur playing a role in the pathogenesis of AFF, such that the anatomy may increase tension on the lateral aspect of the femur [7].

Guidelines advise regular review of OP treatment and to consider drug pause at 5 years if a patient is low to moderate risk. If fracture risk remains high or there is an interval fracture, treatment may be continued for an additional 5 years. Recent guidance from the American Association of Clinical Endocrinologists now suggests consideration of a Bisphosphonate pause after 6 to 10 years of stability in patients with a very high risk of fracture [8]. In most cases such AFFs seldom heal without surgical treatment [9].

Bone is a metabolically active tissue, constantly undergoing repair and remodelling. The bone turnover markers (BTMs), ß-CTX and P1NP are fragments of the major bone structural protein, type 1 collagen. BCTX (resorption marker) is released on degradation of bone collagen matrix and P1NP (formation marker) is produced by osteoblasts during the synthesis of type 1 collagen. In health, resorption and formation rates are tightly linked so that bone mass is unchanged [10]. Measurement of BTMs provides a quantitative estimate of current bone metabolism [11].

An increase in bone turnover is seen with ageing and OP. At menopause, P1NP and B-CTX levels increase and these higher levels are associated with more rapid bone loss. Evidence for the use of BTMs as predictors of fracture is not robust [12] with some studies demonstrating that BTMs levels increase in proportion to fracture risk independent of bone mineral density [13, 14] and others not [15, 16]. The effect of osteoporosis therapy on BTMs depends upon the mechanism of action of the specific therapy. Bisphosphonates cause a rapid decrease in markers of bone resorption, followed by a decrease in bone formation markers [11] while, anabolic agents (e.g.Teriparatide) result in a rapid increase in bone formation markers, followed by an increase in markers of bone resorption. The BTMs in this woman most likely reflect adherence/response to antiresorptive medication as P1NP levels were midpoint of the reference interval while B-CTX concentrations were juxta the lower reference limit.

The medical history of a significant background of early menopause, prolonged Phenobarbitone (50 years approximately) therapy, suboptimal vitamin D levels and residual left hypertonia were contributory factors. In the 1970s, it was reported that vitamin $\mathrm{D}$ was metabolized at a faster rate in patients chronically treated with Phenobarbitone [17], with a mechanism of rapid metabolism mediated by increased hepatic hydroxylation activity[18]. Phenobarbitone is a potent inducer of hepatic cytochrome P450 enzymes including CYP3A4 and CYP24A1 which enhances oxidation of $25(\mathrm{OH}) \mathrm{D}$ and its active hormone, $1,25(\mathrm{OH})_{2} \mathrm{D}$ to their respective physiologically inactive 24-hydroxylated products, $24,25(\mathrm{OH})_{2} \mathrm{D}$ and $1,24,25(\mathrm{OH})_{3} \mathrm{D}$, with resultant metabolic bone disease [19]. Withdrawal of this drug was considered in this lady. However, given a history of severe epilepsy and that she had been seizure-free for decades together with the literature on withdrawal symptoms including intractable withdrawal seizures, a clinical decision was made to continue her current regimen [20].

This lady's Vitamin D level at $65 \mathrm{nmol} / \mathrm{L}$, while within the therapeutic range based on some international guidelines (Institute of Medicine-IOM) [21], was probably suboptimal given that she had severe prolonged $\mathrm{OP}$, was on a medication which markedly enhances vitamin $\mathrm{D}$ degradation which could not be discontinued. 
Thus the higher vitamin 25(OH)D target of $>75 \mathrm{nmol} / \mathrm{L}$ recommended by the Endocrine Society(ENDO) would be more appropriate for this patient [22].

This lady had significant left lower limb hypertonia and hyperreflexia. There is data that suggests an association between contractures related to immobility and increased likelihood of spontaneous fractures, as highlighted by the authors' report of a centenarian with severe contractures who presented with a multifactorial spontaneous fracture of the lower femur [23]. Stroke is a risk factor for fracture for a multitude of reasons, with fractures most likely to occur in the hypertonic or hemiplegic limb [24]. The MEDOS study highlighted a higher risk of hip fracture in patients with a history of stroke with hemiplegia than those without residual hemiplegia [25].

Following surgery, teriparatide was initiated for bone protection with escalation of Vitamin D dosage. Amid uncertainty regarding how to treat patients after they have sustained an AFF and given that bone agents have potential positive and negative effects on fracture healing, recent recommendations, not supported by randomised data, from the European Calcified Tissue Society describe probable beneficial effects on healing time of surgically treated AFFs with teriparatide, although non-union at one year can still occur [5].

This patient was on Bisphosphonate therapy continuously for 17 years and algorithms utilising validated assessments of fracture risk in treated individuals such as Frax $\AA$ are available [26]. Electronic reminders on clinical systems at a 5 -year interval following medication commencement should be trialled. Medication management applications are now available for patients online which can provide reminders following completion of a specified duration of treatment [27]. Such initiatives should assist in reducing excessive duration of Bisphosphonate treatment which could in turn reduce the prevalence of AFFs.

Spontaneous fractures are rare and multifactorial with AFFs representing the most frequent subgroup of patients with fracture of weakened bone due to physiological stress. Careful management of OP by limiting treatment duration with antiresorptive agents, careful attention to other risk factors including adequate vitamin D supplementation and review of culprit medications would all minimise their occurrence.

References

1. Viceconti M, Taddei F, Cristofolini L, Martelli S, Falcinelli C, Schileo E. Are spontaneous fractures possible? An example of clinical application for personalised, multiscale neuro-musculo-skeletal modelling. J Biomech 2012;45:421-6.

2. Lewiecki EM. Bisphosphonates for the treatment of osteoporosis: insights for clinicians. Ther Adv Chronic Dis 2010;1:115-28.

3. Liu J, Zhang HX, Lu XX, Hu JJ, Deng LF. Bisphosphonates and risk of subtrochanteric, femoral shaft, and atypical femur fracture: sensitivity and trim and fill studies. Genet Test Mol Biomarkers 2014;18:117-22.

4. Donnelly E, Saleh A, Unnanuntana A, Lane JM. Atypical femoral fractures: epidemiology, etiology, and patient management. Curr Opin Support Palliat Care 2012;6:348-54.

5. van de Laarschot DM, McKenna MJ, Abrahamsen B, Langdahl B, Cohen-Solal M, Guanabens N, et al. Medical Management of Patients After Atypical Femur Fractures: a Systematic Review and Recommendations From the European Calcified Tissue Society. J Clin Endocrinol Metab 2020;105.

6. Shane E, Burr D, Ebeling PR, Abrahamsen B, Adler RA, Brown TD, et al. Atypical subtrochanteric and diaphyseal femoral fractures: report of a task force of the American Society for Bone and Mineral Research.J Bone Miner Res 2010;25:2267-94.

7. Adler RA. MANAGEMENT OF ENDOCRINE DISEASE: Atypical femoral fractures: risks and benefits of long-term treatment of osteoporosis with anti-resorptive therapy. Eur J Endocrinol 2018;178:R81-R87.

8. Camacho PM, Petak SM, Binkley N, Diab DL, Eldeiry LS, Farooki A, et al. American Association of Clinical Endocrinologists/American College of Endocrinology Clinical Practice Guidelines for the Diagnosis and 
Treatment of Postmenopausal Osteoporosis- 2020 Update Executive Summary. Endocr Pract 2020;26:564570.

9. Ha YC, Cho MR, Park KH, Kim SY, Koo KH. Is surgery necessary for femoral insufficiency fractures after long-term bisphosphonate therapy? Clin Orthop Relat Res 2010;468:3393-8.

10. Burch J, Rice S, Yang H, Neilson A, Stirk L, Francis R, et al. Systematic review of the use of bone turnover markers for monitoring the response to osteoporosis treatment: the secondary prevention of fractures, and primary prevention of fractures in high-risk groups. Health Technol Assess 2014;18:1-180.

11. Vasikaran S, Eastell R, Bruyere O, Foldes AJ, Garnero P, Griesmacher A, et al. Markers of bone turnover for the prediction of fracture risk and monitoring of osteoporosis treatment: a need for international reference standards. Osteoporos Int 2011;22:391-420.

12. Vilaca T, Gossiel F, Eastell R. Bone Turnover Markers: Use in Fracture Prediction. J Clin Densitom 2017;20:346-352.

13. Garnero P, Hausherr E, Chapuy MC, Marcelli C, Grandjean H, Muller C, et al. Markers of bone resorption predict hip fracture in elderly women: the EPIDOS Prospective Study. J Bone Miner Res 1996;11:1531-8.

14. Garnero P, Sornay-Rendu E, Claustrat B, Delmas PD. Biochemical markers of bone turnover, endogenous hormones and the risk of fractures in postmenopausal women: the OFELY study. J Bone Miner Res 2000;15:1526-36.

15. Johansson H, Oden A, Kanis JA, McCloskey EV, Morris HA, Cooper C, et al. A meta-analysis of reference markers of bone turnover for prediction of fracture. Calcif Tissue Int 2014;94:560-7.

16. Tian A, Ma J, Feng K, Liu Z, Chen L, Jia H, et al. Reference markers of bone turnover for prediction of fracture: a meta-analysis. J Orthop Surg Res 2019;14:68.

17. Hahn TJ, Hendin BA, Scharp CR, Haddad JG, Jr. Effect of chronic anticonvulsant therapy on serum 25-hydroxycalciferol levels in adults. N Engl J Med 1972;287:900-4.

18. Hahn TJ, Birge SJ, Scharp CR, Avioli LV. Phenobarbital-induced alterations in vitamin D metabolism. J Clin Invest 1972;51:741-8.

19. Lee RH, Lyles KW, Colon-Emeric C. A review of the effect of anticonvulsant medications on bone mineral density and fracture risk. Am J Geriatr Pharmacother 2010;8:34-46.

20. Lossius MI, Alfstad KA, Aaberg KM, Nakken KO. Discontinuation of antiepileptic drugs in seizure-free patients - when and how? Tidsskr Nor Laegeforen 2017;137:451-454.

21. Ross AC, Manson JE, Abrams SA, Aloia JF, Brannon PM, Clinton SK, et al. The 2011 report on dietary reference intakes for calcium and vitamin D from the Institute of Medicine: what clinicians need to know. J Clin Endocrinol Metab 2011;96:53-8.

22. Holick MF, Binkley NC, Bischoff-Ferrari HA, Gordon CM, Hanley DA, Heaney RP, et al. Evaluation, treatment, and prevention of vitamin D deficiency: an Endocrine Society clinical practice guideline. J Clin Endocrinol Metab 2011;96:1911-30.

23. Brennan M, O'Shea PM, O'Keeffe ST, Mulkerrin EC. Spontaneous Insufficiency Fractures. J Nutr Health Aging 2019;23:758-760.

24. Chiu KY, Pun WK, Luk KD, Chow SP. A prospective study on hip fractures in patients with previous cerebrovascular accidents.Injury 1992;23:297-9.

25. Kanis J, Johnell O, Gullberg B, Allander E, Elffors L, Ranstam J, et al. Risk factors for hip fracture in men from southern Europe: the MEDOS study. Mediterranean Osteoporosis Study. Osteoporos Int 1999;9:45-54. 
26. MHRA. Medicines and Healthcare Products Regulatory Agency. Drug Safety Update:Bisphosphonates (2014): Available at: https://www.gov.uk/government/publications/bisphosphonates-use-and-safety. Accessed: 24 May 2020.

27. Tabi K, Randhawa AS, Choi F, Mithani Z, Albers F, Schnieder M, et al. Mobile Apps for Medication Management: Review and Analysis.JMIR Mhealth Uhealth 2019;7:e13608.

Figure 1. Angulated fracture of upper third of left femur. 